\title{
Determining Query Readiness for Structured Data
}

\author{
Farid Alborzi, Rada Chirkova, Jon Doyle, and Yahya Fathi \\ Computer Science Department, North Carolina State University \\ North Carolina, USA \\ \{falborz, rychirko, jon_doyle,fathi\}@ncsu.edu
}

\begin{abstract}
The quality and character of organizational decisions depends both on the character of the data available to make these decisions and on the value of the data in the decision-making process. This study introduces and investigates Data Readiness Level (DRL) as a quantitative measure of the value of a piece of data at a given point in a processing flow. DRL is considered as a multidimensional measure that takes into account relevance, completeness, and utility of data in confronting tasks. This study provides a formalization of some simple DRL measures and illustrates how to use knowledge of rules and facts about a database and the world to identify transformations of the data that improve DRL.
\end{abstract}

Keywords: data readiness level, data quality measurement, data quality improvement, knowledge base, relational database

\section{Introduction}

\subsection{Motivation}

Data-driven organizations employ analytics in recognizing events of interest, predicting future events, and prescribing actions to be taken. Analytics work by converting input data into information and knowledge. Thus, the input data affect the extracted knowledge and respectively influence the decision-making process. A way to view this is that every piece of data contains an inherent "quality value" which indicates the contribution value of this datum with respect to each specific decision-making process. A "low-quality" value indicates that the data are not reliable and may lead to inferior decision making. As an example, consider a decision making process which requires data provided by Driving Motor Vehicle (DMV) about drivers and their cars' plate numbers and data recorded by a Closed Circuit Camera System (CCCS) collecting data about cars passing a toll booth. The absence of required information for decision making in DMV and CCCS datasets results a "low-quality" value leading to erroneous decisions. Hereafter, this example is called "Toll Booth example" in this paper.

The problem that we consider in this paper is determining whether the "quality value" of data meets, or at least can be improved to meet, the data-quality expectations of decision makers. We approach this problem by introducing methods 
for quantifying and improving the quality value of data. To address this problem, we introduce and investigate data-readiness level ( $D R L)$, a quantitative measure of the quality value of a piece of data at a given point in a processing flow. As such, the DRL represents a paradigm shift from the qualitative aspect of traditional exploratory data analysis towards a rigorous metrics-based assessment of the quality value of data in various states of readiness. Each decision making process is composed of a sequence of analytical tasks, and each task has some requirements on the quality value of the input data, with regard to returning acceptable-quality information or knowledge for the decision making. The DRL represents the value of a piece of data to an analyst, analytic, or other process, and is expected to change as that datum moves through, is interpreted by, or changed by that process. We consider DRL as a multidimensional measure based on one or more data quality dimensions and task characteristics. In "Toll Booth example", DRL contributes to decision making process by measuring the quality value of DMV and CCCS data in terms of data quality dimensions. Moreover, the DRL value demonstrates the distance between the existing information of the provided data and required information for decision making. Consequently, based on the demonstrated distance, improving solutions are employed to increase the quality value.

In this paper, we consider DRL as a vector of data quality dimensions. Data quality is defined as "fitness for use" in [1] and a data quality dimension is defined as an aspect of data that can be measured to quantify the data quality [2]. Consequently, data quality is a task oriented concept measured by data quality dimensions. This study considers relevance and completeness dimensions as DRL components and tasks requirements. We define relevance dimension of a database for a specific task as the closeness of data content and the required attributes to address the task. This definition is in line with [17]. Furthermore, a certain level of data quality such as completeness is required for each attribute to support the managerial decision making. Completeness dimension is defined as availability of all relevant data to satisfy the user requirements in [4]. Consequently, in this study, we consider the completeness of an attribute for a specific task as the availability of all relevant data for the required attribute to address the task.

While dimensions have been extensively studied in the literature to quantify the data quality value of a database for a specific task and also data cleaning methods have been developed to improve the data quality value of a database,to the best of our knowledge no formalization has been developed for integrating the computation and improvement of the task-dependent data-quality value of a database considering multiple tasks. This study introduces the first approach to such a formalization, for measuring the DRL value that helps determining the quality-value of data, as well as for improving it.

Dimensions in this study are computed with respect to a specific task to contribute in evaluating the overall DRL of a database for multiple tasks. Thus, dimensions are task oriented and require constructing attributes of a task. In the toll booth example, consider as a task that a city's traffic-management department is looking for the owners of all the cars passed the toll booth on a specific 
day and their plate was registered in a specific state. Thus, the task requirements are existence of drivers name, date, and state attributes in the datasets and a certain level of completeness for each attribute. Moreover, existing datasets are different in satisfying the requirements. Although the existence of the required attributes in the datasets and the completeness of each attribute are critical to address the task, attributes might be different in their importance based on the level of their contribution to address the task and satisfy users demand.

This study uses relevance and completeness dimensions to define DRL for a relational database. The foundational fact concerning data readiness is that data-readiness assessments rely on knowledge extracted from the data and in turn constitute knowledge about the data. At the highest level of abstraction, two major goals can be considered for DRL:

1. Determine whether the data have information of sufficient quality with respect to the task.

2. If the answer is negative, identify ways to increase the information quality with respect to the task.

While defining DRL addresses the first goal, it does not provide solutions for the second goal. Identifying readiness-improving operations requires knowledge about the database itself, the task domain, and the task environment.

\subsection{Contribution and Plan of the Paper}

The main contribution of this study is an initial formalization of data readiness level, which includes the following elements.

- Section 3 defines a simple DRL measure for relational databases that combines measures of data completeness and relevance motivated by a simple analytical task.

- Section 4 illustrates how to quantify the effects of data-readying operators of familiar types, and how to integrate data-readiness evaluation with knowledge about the task domain and the database to improve DRL.

- Section 5 explains the concepts introduced in section 4 through employing a use case.

- Section 6 summarizes key points, presents conclusions, and highlights directions for future work.

Section 2 first summarizes related work on quantification and improvement of data quality and the use of such measures in knowledge management.

\section{Related Works}

While there have been many publications defining and classifying data quality dimensions relating data format and syntactic criteria $([5],[6],[7],[8],[9])$, some studies have been investigating the semantics of data values $([3],[10],[12],[11])$. 


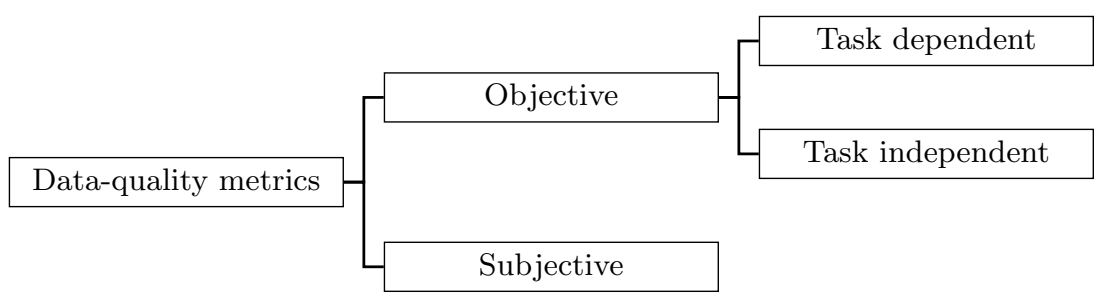

Fig. 1. Data quality metric types

Related studies follow two main streams of measuring data quality: a) quality of conformance and b) quality of design ([13],[14]). Quality of conformance aims the correspondence between information system's existing data values and its design specifications ([13]). Quality of design, however, checks the closeness of system's specifications and customers' requirements ([13]). Consequently, while quality of conformance is mostly objective, quality of design is a subjective concept. While subjective data quality assessment can be approached by distributing questionnaire among stakeholders ([15]), studies published on objective measurement investigate descriptive metrics. Objective assessments can be either task-dependent or task-independent ([15]). Task-independent assessments (or "impartial" as they are called in [16]) do not depend on the task and just give a general view of the data status. In contrast, task-dependent assessments (or "contextual" as they are called in [16]) are designed for a specific application. Figure 1 summarizes the above discussion.

The data quality metrics investigated in the literature either consider one specific task or no task to quantify the data quality. This study, however, provides a framework based on which metrics can be computed considering a collection of tasks imposed on a database.

Researchers have proposed principles and requirements to be enforced on the metrics. Even and Shankaranarayanan ([16]) proposed interpretation consistency, representation consistency, aggregation consistency, and impartial-contextual consistency as consistency rules to be followed by metrics. Moreover, Heinrich et al. ([3]) mentioned normalization, interval scale, interpretability, aggregation, adaptivity, and feasibility as six requirements on metrics. In this study, we consider two metrics: a) relevance, and b) completeness.

Relevance is defined as "the level of consistency between the data content and the area of interest of the user" in [17]. In this study, we define relevance in a similar context as the closeness of data content and the required attributes to address the task. While relevance has been addressed in several studies ([20],[22],[21], [7], [19], [18]) as a data quality dimension, to the best of our knowledge no metric has been provided to quantify it in the literature. The framework proposed in this study provides a structure based on which a metric is formulated to not only compute the current level of relevance, but find solutions to improve it. 
Completeness is defined as a measure of non-null values of an attribute ([7], [15]). We base the same definition for both formulating the completeness of a database and finding solutions to improve it. It is extensively accepted in the literature that existing null values in a database results a deterioration in the quality of the information and misleads the users ([23]). Thus, there have been both practical and theoretical approaches in database management systems literature to handle problems originating from null values existence in a database. In 1970s, Codd ([24],[25]) investigated handling null values in commercial database management systems. [24] and [25] developed a three-valued logic to solve the problem of working with null values and the logic was reflected in SQL standard. On one hand, the practical solutions such as the three-valued logic feature for SQL has been criticized in several studies ([26], [27]), on the other hand, the theoretical approaches have been so complicated hindering them to be used in applications ([28]). There are three main streams of studies in the literature of incomplete information([28]) developing approaches based on: a) representation systems and certain answers([29],[30]); b) logical theory ([31],[32]); and c) programming semantics ([33],[34]). While these studies use the structure contained in the relational databases to work with incomplete information and do not use any external knowledge to improve the completeness, this study develops a formalization to improve completeness based on rules and facts contained in the database and/or provided by user.

Knowledge bases have been progressively used in academia and industry on a variety of applications ([35]). While [36] developed a platform employing user-imposed rules to repair data based on detecting the rules violations, the platform was not established on the quality value evaluation of a database confronting tasks. This study develops a framework measuring the task-dependent quality value of data to support exploiting a knowledge base. The contained knowledge aims to find quality improving solutions and is composed of the facts about the database structure in addition to rules about data quality dimensions improvement.

\section{Formalizing DRL}

This section provides a concrete definition of DRL for the data represented in a relational database and tasks consisting of simple SQL queries. We sketch extension of the formalism to other data representations and more complicated tasks in Section 6.

\subsection{Example 1: Traffic Flow Identification}

Suppose that a city's traffic-management department wants to know the owners of cars registered in North Carolina (NC) that entered the city through a local toll road on July 4, 2014. The toll road operates toll booths equipped with closedcircuit camera systems connected to a license-plate recognition system (LPR). This system identifies the number on a license plate with some possibility of 
Table1 from LPR

\begin{tabular}{|c|c|c|c|c|c|c|}
\hline row & plate & lane & CL & user & time & date \\
\hline \hline 1 & NMU45 & 3 & 1.00 & $\perp$ & $09: 20: 16$ & $07 / 04 / 2014$ \\
\hline 2 & STA00 & 1 & 0.73 & n1 & $09: 20: 03$ & $07 / 04 / 2014$ \\
\hline 3 & ABWD9 & 3 & 0.85 & n2 & $09: 19: 53$ & $07 / 04 / 2014$ \\
\hline 4 & TRC19 & 4 & 1.00 & n3 & $09: 19: 52$ & $07 / 04 / 2014$ \\
\hline
\end{tabular}

Table2 from DMV

\begin{tabular}{|c|c|c|c|}
\hline LN & name & plater & state \\
\hline \hline 11156 & n2 & ABWD9 & VA \\
\hline 78922 & n1 & STA00 & VA \\
\hline 58556 & w1 & NMU45 & NC \\
\hline 82659 & n3 & TRC19 & MD \\
\hline
\end{tabular}

Fig. 2. Relational tables in database $D_{1}$. CL stands for Confidence Level in Table1, and LN stands for License Number in Table2, and $\perp$ stands for a null value.

error, for which the system supplies either a confidence figure in the interval $[0,1]$ or a null value, denoted $\perp$. The city sets 0.8 as the minimum confidence required in answering its query about $\mathrm{NC}$ registrants.

Suppose further that the city's database $D_{1}$ is a relational database that includes copies a table Table1 obtained from the LPR system and a table Table2 obtained from the NC Department of Motor Vehicles (DMV), as presented in Figure 2. Observe that neither Table1 nor Table2 can address the aforementioned task perfectly by itself. The query $Q_{1}$ given by

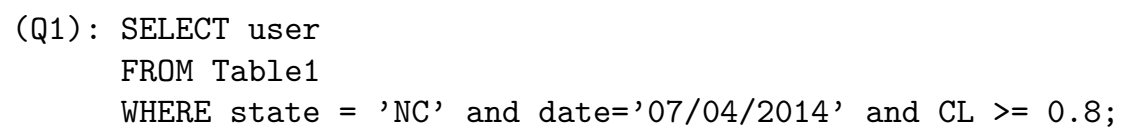

fails because of the lack of the attribute state and the null value in row 1 , and the query $Q_{2}$ given by

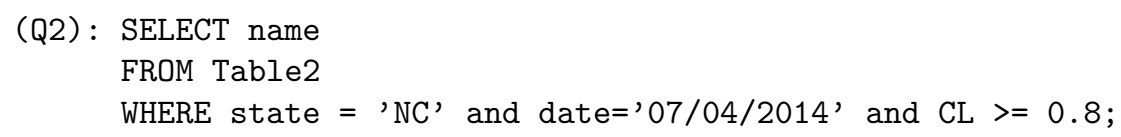

fails because of the lack of the attributes date and CL. Together, however, the two tables can provide the desired answers, as we explain below.

\section{$3.2 \quad$ Tasks}

For simplicity of treatment, the formalization of DRL undertaken here restricts attention to data stored in relational databases, and each task consists of a standard SQL query with a restricted form. More general formalizations drop or weaken these restrictions and broaden the conception of task.

We first elaborate the notion of task used here.

Definition 1. A task is a SQL query composed of SELECT, FROM, and WHERE clauses that has no subqueries and in which the FROM clause references a single table.

For analytical purposes, we abstract away the details of a query and consider only the attributes and tables referenced in the query in what we call a task signature. 
Definition 2. The task signature of a query $Q$, written $T(Q)$, is a triple

$$
T(Q)=[S(Q), F(Q), W(Q)]
$$

in which $S(Q)$ is the set of attributes in the SELECT argument of $Q, F(Q)$ is the set of tables in the FROM argument of $Q$, and $W(Q)$ is the set of attributes in the WHERE argument of $Q$.

In considering of enumerated queries $Q_{1}, \ldots, Q_{n}$, we write $T_{i}, S_{i}, F_{i}$, and $W_{i}$ for the signature elements of $Q_{i}$.

Definition 3. The available attributes of a query $Q$, written $A(Q)$, is the set of all attributes in all tables in $F(Q)$.

Of course, restricting attention to queries with only one FROM table means that the available attributes of a query are just the attributes in that single table.

For example, the queries $Q_{1}$ and $Q_{2}$ given in Example 1 have the following signatures.

$$
\begin{aligned}
& T_{1}=[\{\text { user }\},\{\text { Table } 1\},\{\text { state }, \text { date }, \mathrm{CL}\}] \\
& \left.T_{2}=[\{\text { name }\},\{\text { Table } 2\}, \text { state }, \text { date }, \mathrm{CL}\}\right]
\end{aligned}
$$

The available attributes of these queries consist of the following sets.

$$
\begin{aligned}
& A_{1}=\{\text { row, plate }, \text { lane }, \mathrm{CL}, \text { user }, \text { time }, \text { date }\} \\
& A_{2}=\{\mathrm{LN}, \text { name }, \text { plate }, \text { state }\}
\end{aligned}
$$

As noted previously, the SELECT and WHERE attributes of these queries refer to attributes not among the attributes available in the query.

For convenience, we sometimes include the set of available attributes in the query signature, writing $T=[S, F, W, A]$.

\subsection{Database Relevance Utility}

We regard readiness judgments as involving notions of utility of data in performing tasks. Utility might depend on numerous factors, but for this initial study we consider a very simple form of utility that assumes readiness depends only on the signature of a task, not on the specific query, and that combines a notion of relative relevance of different attributes in the task signature with a notion of relative importance of completeness of different attributes in the task signature.

Definition 4. The task-specific relevance utility of an attribute to a task signature $T=[S, F, W]$, denoted $\rho(a, T)$ for $a \in S$ or $a \in W$, is a nonnegative number representing the relative importance of that attribute in performing the task.

We regard these attribute utilities as cardinal utilities, that is, $\rho(a, T)=2 \rho\left(a^{\prime}, T\right)$ means that the relevance of $a$ is twice as important to readiness as is $a^{\prime}$.

We combine these attribute utilities to obtain a measure of the relevance utility of an entire database to a task. 


\begin{tabular}{|c|c|c|c|c|}
\hline$a$ & user & state & date & CL \\
\hline$\rho(a)$ & 4 & 1 & 4 & 2 \\
\hline
\end{tabular}

Table 1. Relevance utility values for $Q_{1}$.

Definition 5. The relevance utility of a database $D$ for a task with signature $T=[S, F, W, A]$, denoted $R(D, T)$, is defined as the proportion of the total relevance utility of the task attributes available in the database (available relevance utility) to the total relevance utility of all the attributes in the task (ideal relevance utility), or formally,

$$
R(D, T)= \begin{cases}\frac{\sum_{a \in S \cap A} \rho(a, T)+\sum_{a \in W \cap A} \rho(a, T)}{\sum_{a \in S} \rho(a, T)+\sum_{a \in W} \rho(a, T)} & \text { if D contains } F \\ 0 & \text { otherwise. }\end{cases}
$$

By $D$ contains $F$, we mean that the database $D$ includes each of the tables in $F$. The numerator of the fraction in 4 represents available relevance utility and the denominator represents ideal relevance utility. Thus if the query FROM table exists in the database, a weighted average method is used to formulate the relevance of the database to the task, while if the FROM table does not exist in the database, the corresponding relevance value is zero.

To extend Example 1, assume that the relative relevance utilities of attributes for $T_{1}$ are given by those in Table 1 . Also, assume that the relevance utility of attributes for $T_{2}$ are the same as those in Table 1. Moreover, consider name and user attributes in $T_{1}$ and $T_{2}$ respectively, having the same relevance utility value. Using (4), we compute the relevance of $D_{1}$ for $T_{1}$ and $T_{2}$ using the signatures given in (2) and (3) to be

$$
\begin{aligned}
R\left(D_{1}, T_{1}\right) & =R\left(D_{1},[\{\text { user }\},\{\text { Table } 1\},\{\text { state }, \text { date }, \text { CL }\}]\right) \\
& =\frac{4+0+4+2}{4+1+4+2} \\
& =0.91 \\
R\left(D_{1}, T_{2}\right) & =R\left(D_{1},[\{\text { name }\},\{\text { Table } 2\},\{\text { state }, \text { date }, \text { CL }\}]\right) \\
& =\frac{4+1+0+0}{4+1+4+2} \\
& =0.45
\end{aligned}
$$

\subsection{Database Completeness Utility}

In order to define the completeness utility of a database for a task, we first define the notion of attribute completeness for a database as follows. We write $r \in D$ to mean that the relational database $D$ contains a relational table $r$. We write $a \in r$ to mean that the relation $r$ contains an attribute $a$. When $r \in D$, we write $|r|$ to denote the number of rows in $r$, and when $a \in r$ as well, write $|r(a \neq \perp)|$ to denote the number of rows in $r$ in which a nonnull value appears for $a$. 


\begin{tabular}{|c|c|c|c|c|}
\hline$a$ & user & state & dater & CL \\
\hline$\kappa(a)$ & 1 & 2 & 4 & 3 \\
\hline
\end{tabular}

Table 2. Completeness utility values for $Q_{1}$ of Example 1 .

Definition 6. The attribute completeness fraction of an attribute a with respect to a database relation $r$, denoted $\phi(a, r)$, is defined as the fraction of rows of $r$ containing non-null values for a, or formally,

$$
\phi(a, r)= \begin{cases}\frac{|r(a \neq \perp)|}{|r|} & \text { if } a \in r \text { and }|r| \neq 0 \\ 0 & \text { otherwise. }\end{cases}
$$

For example, in Example 1, taking $r$ to be Table1, $\phi($ time, $r)$ is 1, while $\phi($ user, $r)$ is $\frac{3}{4}$.

We now employ a notion of relative utility of the completeness of attributes, paralleling the notion of attribute relevance utilities.

Definition 7. The task-specific completeness utility of an attribute to a task signature $T=[S, F, W]$, denoted $\kappa(a, T)$ for $a \in S$ or $a \in W$, is a nonnegative number representing the relative importance of the completeness of that attribute to performing the task.

We assume these completeness utility values are cardinal and depend only on the task signature.

One can now combine attribute completeness utilities into a database completeness utility as follows.

Definition 8. The database completeness utility of a database $D$ for a task with signature $T=[S, F, W, A]$, denoted $K(D, T)$, is defined as the proportion of the total completeness utility of the task attributes available in the database (available completeness utility) to the total completeness utility of all the attributes in the task (ideal completeness utility), or formally,

$$
K(D, T)= \begin{cases}\frac{\sum_{a \in S} \kappa(a, T) \phi(a, F)+\sum_{a \in W} \kappa(a, T) \phi(a, F)}{\sum_{a \in S \cap A} \kappa(a, T)+\sum_{a \in W \cap A} \kappa(a, T)} & \text { if } D \text { contains } F \\ 0 & \text { otherwise. }\end{cases}
$$

The completeness utility of a query is computed as the completeness utility of each query attribute weighted by its completeness (the numerator in (6)), and normalized by the total possible completeness utility (the denominator in (6)).

To extend Example 1 further, assume that the completeness utilities of attributes for both $T_{1}$ and $T_{2}$ are given by those in Table 2. Also, consider name and user attributes in $T_{1}$ and $T_{2}$ respectively, having the same completeness 
utility value. Using (5) and (6), we compute the completeness utility of $D_{1}$ for $T_{1}$ and $T_{2}$ using the signatures given in (2) and (3) to be

$$
\begin{aligned}
K\left(D_{1}, T_{1}\right) & =K\left(D_{1},[\{\text { user }\},\{\text { Table } 1\},\{\text { stater, date }, \text { CL }\}]\right) \\
& =\frac{1 .\left(\frac{3}{4}\right)+2 .(0)+4 .(1)+3 .(1)}{1 .(1)+2 .(0)+4 .(1)+3 .(1)} \\
& =0.97 \\
K\left(D_{1}, T_{2}\right) & =K\left(D_{1},[\{\text { name }\},\{\text { Table } 2\},\{\text { state }, \text { date }, \text { CL }\}]\right) \\
& =\frac{1 .(1)+2 .(1)+4 .(0)+3 .(0)}{1 .(1)+2 .(1)+4 .(0)+3 .(0)} \\
& =1
\end{aligned}
$$

\subsection{Database DRL}

Based on the discussions in Sections 1 and 2 we define data readiness level as a vector of relevance and completeness dimensions in which relevance represents the closeness between data content and task requirements, and in which completeness represents availability of values for attributes required to address a task.

Definition 9. The data readiness level (DRL) of a database $D$ with respect to a task $T$, written $D R L(D, T)$, consists of the relevance and completeness utilities of the database with respect to the task, namely

$$
\operatorname{DRL}(D, T)=[R(D, T), K(D, T)] .
$$

For example, in Example 1 the DRL for $D_{1}$ for $T_{1}$ and for $T_{2}$ are given by

$$
\begin{aligned}
& \operatorname{DRL}\left(D_{1}, T_{1}\right)=[0.91,0.97] \\
& \operatorname{DRL}\left(D_{1}, T_{2}\right)=[0.45,1] .
\end{aligned}
$$

To obtain a unidimensional measure of readiness, one can instead regard relevance and completeness utilities as subutility functions of a more inclusive conception of utility of a database for a task. To do this, one defines a total utility function $D R L^{*}(D, T)$ taking numerical values. Such a total utility function might be obtained as some function of the relevance and completeness utility figures alone, such as a weighted sum of the two $\left(w_{1} R(D, T)+w_{2} K(D, T)\right.$, or as a nonlinear combination of the two components, or as a more general evaluation that takes into account other properties of the database and task.

To extend the notion of database readiness to several tasks, one can average readiness across tasks or treat readiness as a game-theoretic multicriteria optimization problem.

In the task averaging approach, one identifies an importance or weight or probability measure over the set $\left\{Q_{1}, \ldots, Q_{I}\right\}$ of possible queries, with $w(Q)$ 
denoting the weight of $Q$, and obtains a weight-relative measure of DRL as

$$
D R L(D)=\frac{\sum_{i=1}^{I} w\left(Q_{i}\right) D R L\left(D, T_{i}\right)}{\sum_{i=1}^{I} w\left(Q_{i}\right)} .
$$

Here one multiplies and sums readiness and completeness components pointwise.

For example, one might weight each query by the number of times it is imposed on the database. Using this interpretation in further extending Example 1 , assume that $Q_{1}$ is performed 50 times on $D_{1}$, and that $Q_{2}$ is performed 100 times. The averaging approach then yields an overall DRL for $D_{1}$ of

$$
\operatorname{DRL}\left(D_{1}\right)=\frac{50 .(0.91,0.97)+100 .(0.45,1)}{150}=[0.60,0.99] .
$$

The multicriteria optimization approach comes in two versions. In the pessimistic optimization, one seeks to minimize the worst one can do with respect to the two readiness dimensions, seeking to identify the elements of

$$
\min _{i}\left(D R L\left(D, T_{i}\right)\right)
$$

In the optimistic version, one seeks instead to maximize the readiness dimensions as represented by the elements of

$$
\max _{i}\left(D R L\left(D, T_{i}\right)\right) .
$$

Identifying these solutions constitutes a Multi Criteria Decision Making (MCDM) problem, and more specifically, a Multi Attribute Decision Making (MADM) problem, which can be solved by various solution approaches (TOPSIS, AHP, SAW, etc.).

Applying the multicriterion optimization approach to Example 1 under the same frequency assumptions as considered before, one sees that $D R L\left(D_{1}, T_{1}\right)$ is higher in relevance but lower in completeness than $D R L\left(D_{1}, T_{2}\right)$. Thus in the absence of further optimization criteria, $D_{1}$ is Pareto optimal with respect to the two queries.

\section{Improving DRL}

Section 3 defined the DRL of a database can be computed both for a specific task and for multiple tasks, and used Example 1 to illustrate how these definitions do not by themselves provide the decision maker with means for improving an unsatisfactory DRL. In this section, we examine the problem of improving DRL by assessing the effects on DRL of a variety of operations on databases called Data-Readying Operators (DROs) that find and fix the flaws and deficiencies in data in respect to imposed task in a timely manner. 


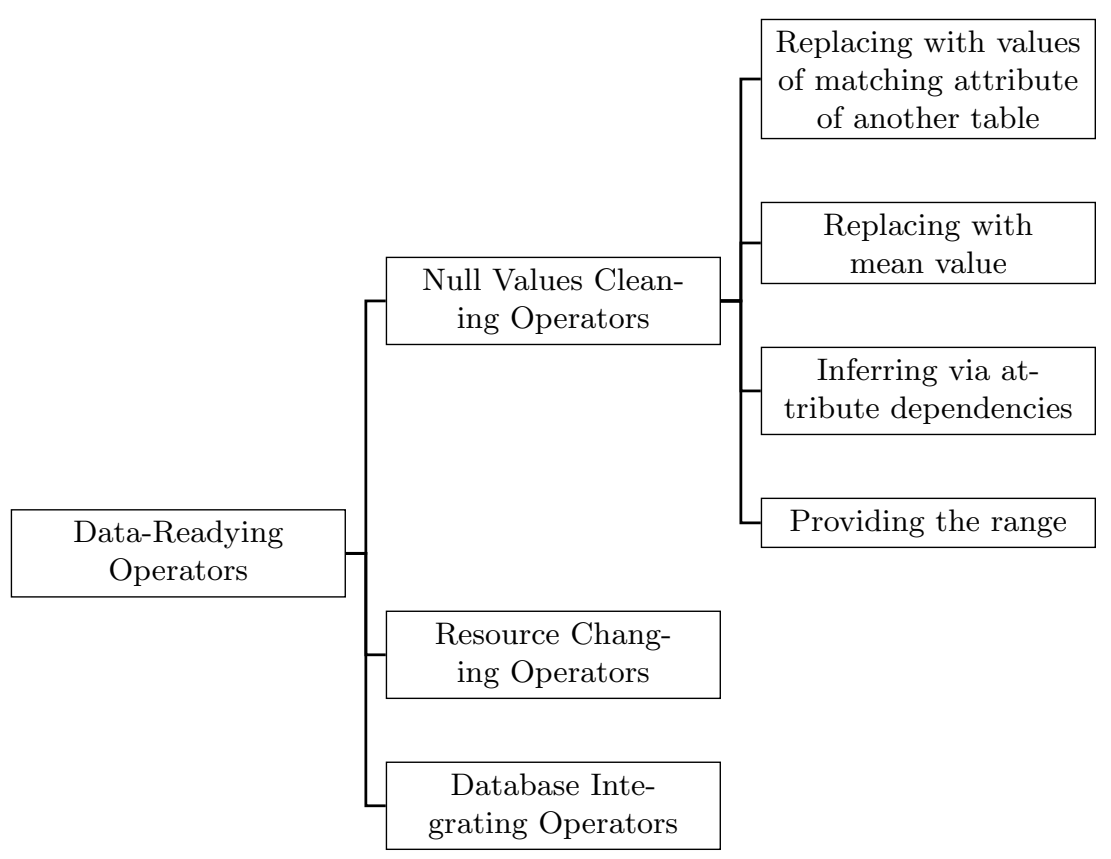

Fig. 3. Data-Readying Operator types

\subsection{Data-Readying Operators}

Operators aimed at improving data readiness fall into three main classes: Null Values Cleaning Operator (NVCO), Resource Changing Operator (RCO), and Database Integrating Operator (DIO). Figure 3 presents these as part of a taxonomy of operators. NVCOs look for null values existing in the relations of a database and uses four possible techniques to clean the null values which increases the completeness of the database. RCOs look for alternative resources (relations) for a task to increase the relevance of the database for that specific task. Finally, DIOs look for appropriate databases which can be integrated with current database to improve both relevance and completeness. Since DIOs require specific assumptions beyond the ones we considered in Section 3 of this paper, they are not detailed here.

A knowledge base containing knowledge about database and external knowledge provided by user is required to implement the DROs. In this section, we introduce DROs and explain how they can be used to improve DRL. All of the operators explained in this section require logical statements in terms of rules describing how the DRO operates. Moreover, the knowledge base must contain facts about database structure such as the name and the schema of existing relations to support the DROs. Furthermore, the additional requirements for implementing each DRO are explained in the following. 


\begin{tabular}{|c|c|c|c|c|c|c|}
\hline row & plater & lane & CL & user & time & date \\
\hline 1 & NMU45 & 3 & 1.00 & w1 & $09: 20: 16$ & $07 / 01 / 2014$ \\
\hline 2 & STA00 & 1 & 0.73 & n1 & $09: 20: 03$ & $07 / 01 / 2014$ \\
\hline 3 & ABWD9 & 3 & 0.85 & n2 & $09: 19: 53$ & $07 / 01 / 2014$ \\
\hline 4 & TRC19 & 4 & 1.00 & n3 & $09: 19: 52$ & $07 / 01 / 2014$ \\
\hline
\end{tabular}

Table 3. Improved Table1 of LPR data

\subsection{Null Values Cleaning Operators}

NVCOs aim to improve DRL by removing null values. NVCOs are explained in the following:

Matching Attributes This method aims to clean null values of an attribute in a table using non-null values of a matching attribute in another table. Two attributes are matched if they represent the same concept in the subject matter ontology. Cleaning the null values can be done by using a concept appearing in both tables as a base for pairing records across tables.

For example, in Example 1, Table1 has attributes plate and user pointing to concepts PlateNumber and UserName, also Table2 has attributes plate and name pointing to concepts PlateNumber and UserName. Thus, plate in Table1 matches plate in Table2 and user in Table1 matches name in Table2. Consequently, null values of user attribute in Table1 can be replaced by name attribute of Table2 based on the concept PlateNumber and through pairing the records containing the same values of plate attribute in Table1 and plate attribute in Table2. The output of implementing this matching attribute DRO on user attribute of Table1 in Example 1 is demonstrated in Table 3.

In addition to knowledge about database structure and the operator functionality, implementing attribute matching NVCO requires several types of knowledge, namely:

- A subject-matter ontology containing the concepts present in the database; and

- A mapping between equivalent concepts and table attributes.

Replacement With the Mean In some applications, knowledge of statistical characteristics of a numerical attribute is used to clean null values. In particular, if the mean and distribution type of the values of an attribute are known, or are known to be close to the mean of the non-null values of the attribute in the database, one can replace null values by the mean value.

For example, in Example 1, if the confidence level of generated plate numbers in the LPR system is normally distributed and there are enough observations in the relation to identify the mean and variance with some accuracy, then one cleaning strategy can be replacing the null values of CL attribute with the average of existing CL values. 
In addition to knowledge about database structure and the operator functionality, implementing replacement with the mean value requires statistical knowledge about the distribution of attributes.

Inferring Values Through Attribute Dependencies This approach attempts to infer missing values of an attribute from dependencies that relate the missing values to values of other attributes.

Implementing value inference requires knowledge of the dependencies among different attributes in addition to knowledge about database structure and the operator performing behavior.

Evaluating the Range of Null Values This DRO aims to provide a range for the values of an attribute. If it is assumed that a continuous quantitative attribute has normal distribution, then about 99.74 percent of attribute values fall into \pm 3 standard deviation from mean value. Thus, if the number of non-null values for the attribute is statistically adequate, then this DRO provides a range for null values by computing the mean and standard deviation logically. The idea is by $99.74 \%$ confidence level the null value falls in $\mu \pm 3 \sigma$.

In addition to general requirements of knowledge about database structure and the operator functionality, this DRO operates through the statistical knowledge about distribution of attributes.

\subsection{Resource Changing Operators}

This DRO changes the FROM argument of queries to see if the new table or tables yields a better DRL value than the old table or tables. If it does, the query is changed to reflect the new FROM argument.

In addition to general requirements, this DRO requires the following for implementation:

- Logical definition of DRL which is able to compute the completeness and relevance logically; and

- A module to find available resources or relations to replace the FROM argument of the task.

\section{Use Case: Marketing}

To demonstrate the concepts introduced in section 4, we introduce the following use case to complement Example 1. In this use case, the marketing department of a company decides to mail customers over 20 years old with Plan B to motivate them to switch to Plan A.

The relational database $D_{2}$ contains the Sales and Customer relations presented in Figure 4. These contain a company's information about its sales and customers.

To answer the marketer's question, one asks the following query: 
Table4: Sales

\begin{tabular}{|c|c|c|c|}
\hline id & customerId & boughtPlan & date \\
\hline 1 & 103 & Plan B & $01 / 11 / 2015$ \\
\hline 2 & 102 & Plan B & $10 / 05 / 2014$ \\
\hline 3 & 104 & Plan A & $12 / 28 / 2014$ \\
\hline 4 & 102 & Plan A & $01 / 07 / 2014$ \\
\hline
\end{tabular}

Table5: Customer

\begin{tabular}{|c|c|c|c|c|}
\hline id & name & address & currentPlan & age \\
\hline 101 & David Smith & 22nd St. & Plan A & $\perp$ \\
\hline 102 & Alfred Luck & 20th St. & $\perp$ & 18 \\
\hline 103 & Daniel Bush & 25th St. & Plan B & 30 \\
\hline 104 & Goldy Elbetri & 7th St. & $\perp$ & $\perp$ \\
\hline
\end{tabular}

Fig. 4. Sales and Customer tables for Marketing problem

\begin{tabular}{|c|c|c|c|c|}
\hline$a$ & name & address & currentPlan & age \\
\hline$\kappa(a)$ & 1 & 4 & 4 & 2 \\
\hline
\end{tabular}

Table 4. Completeness utilities for Example 2

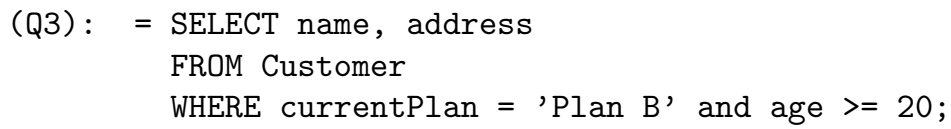

Consequently, $T_{3}$ is as follows:

$$
T_{3}=[\{\text { name }, \text { address }\},\{\text { Customer }\},\{\text { currentPlan, age }\}] .
$$

Since all of the attributes required to address $Q_{3}$ are contained in the database, the relevance value of database $D_{2}$ for $T_{3}$ is 1 . Assume that the completeness utilities for attributes in $T_{3}$ are contained in Table 5. Following the formulas in Section 3 yields $\operatorname{DRL}\left(D_{2}, T_{3}\right)=[1.0,0.73]$.

Assuming that the values of the age attribute in Customer table are normally distributed, there are statistically enough observations with non-null value for age attribute in Customer table, and most of the observations on customers fall around mean value, replacing the null values of age attribute with its mean value is possible. The output of implementing this DRO in Marketing problem on age attribute of Customer table is demonstrated in Table 5.

Moreover, assume that we know whenever the value of currentPlan for a customer is null, it can be replaced with the latest plan that customer bought. The corresponding DRO looks for tuples in Sales table with the same customerId as the id attribute in Customer table with null values of currentPlan attribute. After finding the related tuples in Sales table, this DRO finds the most recent transaction for the customer by finding the maximum value of date attribute. Next, it asserts a new tuple in Customer table which contains the most recent boughtPlan value for currentPlan attribute value and retracts the tuple with null value. The output of implementing this DRO in Marketing problem on currentPlan attribute of Customer table is demonstrated in Table 5. 


\begin{tabular}{|c|c|c|c|c|}
\hline id & name & address & currentPlan & age \\
\hline 101 & David Smith & 22nd St. & Plan A & 24 \\
\hline 102 & Alfred Luck & 20th St. & Plan B & 18 \\
\hline 103 & Daniel Bush & 25th St. & Plan B & 30 \\
\hline 104 & Goldy Elbetri & 7th St. & Plan A & 24 \\
\hline
\end{tabular}

Table 5. Improved Table5 of Customer table

\section{Conclusion and Future Work}

This paper has studied the problem of quantifying the readiness of a relational database to handling tasks expressed as SQL queries. We call this the Data Readiness Level or DRL of the database with respect to the task.

We formalized the problem to not only evaluate the current level of data readiness in terms of relevance and completeness data quality metrics, but also to identify "data readying" operators that improve readiness. The proposed formalization is extensible to quantify the overall relevance and completeness data quality dimensions of a database with respect to a set of new tasks or queries. We discussed several DRL-improving operations and identified the knowledge required to apply them as including a) a structure mapping attributes to concepts in the database ontology to find matching attributes in the relations, b) the logical definition of data readiness level, c) logical definition of data readying operators, d) facts about relations in the database, and e) the rules provided by user about the world in which the database exists and the attribute dependencies.

This inital study of DRL involves several simplifying assumptions about the structure of databases, tasks, and task-dependent utility of different representations of data. Our future work aims to relax these assumptions, and this aim provides many important problems to investigate. Specifically, considering inference tasks that employ more than one table permits DRL assessment and improvement to involve joined relations. Extending the framework to allow subqueries enables treatment more complex and realistic scenarios. Finally, data readiness assessment can be extended to cover additional data quality dimensions and application to databases other than relational databases.

Acknowledgment. This material is based upon work supported in whole or in part with funding from the Laboratory for Analytic Sciences (LAS). Any opinions, findings, conclusions, or recommendations expressed in this material are those of the author(s) and do not necessarily reflect the views of the LAS and/or any agency or entity of the United States Government.

\section{References}

1. Lee, Y.W., Pipino, L.L, Funk, J.D., Wang, R.Y. : Journey to Data Quality The MIT Press, London, 67108, (2006). 
2. Sebastian-Coleman, L.: Measuring data quality for ongoing improvement a data quality assessment framework. Burlington: Elsevier Science, 40-53, ch04, (2013).

3. Heinrich, B., Kaiser, M., Klier, M.: How to measure data quality? A metric-based approach (2007)

4. Gardyn, E.: A Data Quality Handbook for a Data Warehouse. Infrastructure. IQ, 267-290 (1997)

5. Wang, R. Y., Storey, V. C., Firth, C. P.: A framework for analysis of data quality research. Knowledge and Data Engineering, IEEE Transactions on, 7(4):623-640, (1995).

6. Redman, T. C., Blanton A.: Data quality for the information age. Artech House, Inc., (1997).

7. Lee, Y. W., Strong, D. M., Kahn, B. K., Wang, R. Y.: Aimq: a methodology for information quality assessment. Information \& management, 40(2) 133-146, (2002).

8. Eppler, M. J.: Managing information quality: increasing the value of information in knowledge-intensive products and processes. Springer, (2006).

9. English, L. P.: Improving data warehouse and business information quality. J. Wiley \& Sons, (1999).

10. Hinrichs, H., Datenqualitatsmanagement in Data Warehouse-Systemen. PhD thesis, Universitat Oldenburg, (2002).

11. Heinrich, B., Klier, M., Kaiser, M., A procedure to develop metrics for currency and its application in crm. Journal of Data and Information Quality (JDIQ), 1(1):5, (2009).

12. Ballou, D., Wang, R., Pazer, H., Tayi, G., K.: Modeling information manufacturing systems to determine information product quality. Management Science, 44(4):462484, (1998).

13. Heinrich, B., Helfert, M.: Analyzing data quality investments in crm. a model-based approach. (2003).

14. Teboul, J.: Managing quality dynamics. Prentice Hall, (1991).

15. Pipino, L. L., Lee, Y. W., Wang, R. Y.: Data quality assessment. Communications of the ACM, 45(4):211-218, (2002).

16. Even, A., Shankaranarayanan, G., Utility-driven assessment of data quality. ACM SIGMIS Database, 38(2):75-93, (2007).

17. Kulikowski, J. L.: Data Quality Assessment. In: Ferraggine, V. E., Doorn, J. H., Rivero, L. C. : Handbook of Research on Innovations in Database Technologies and Applications: Current and Future Trends (2 Volumes). pp. 378-384 Hershey, PA: Information Science Reference. doi:10.4018/978-1-60566-242-8. ch041 (2009).

18. Wang, R. Y., Strong, D. M.: Beyond accuracy: what data quality means to data consumers. Journal of Management Information Systems 12 (4), pp. 534, (1996).

19. Jarke, M., Vassiliou, Y.: Data warehouse quality: a review of the DWQ project. Proceedings of the Conference on Information Quality, Cambridge, MA, pp. 299313, (1997).

20. Delone, W. H., McLean, E. R.: Information systems success: the quest for the dependent variable. Information systems research 3 (1), pp. 6095 (1992).

21. Redman, T. C.: Data Quality: Management and Technology. Bantam Books, New York, NY, (1992).

22. Wand, Y., Wang, R. Y.: Anchoring data quality dimensions in ontological foundations Communications of the ACM 39 (11), pp. 86-95 (1996).

23. Date C. J., Database in Depth - Relational Theory for Practitioners. OReilly, 2005.

24. Codd, E. F.: Understanding relations (installment \# 7). FDT - Bulletin of ACM SIGMOD, 7(3) 2328, (1975). 
25. Codd, E. F.: Extending the database relational model to capture more meaning. ACM TODS, 4(4) 397434, (1979).

26. Date, C. J.: Database in Depth - Relational Theory for Practitioners. OReilly, (2005).

27. Date, C. J., Darwin, H.: A Guide to the SQL Standard. Addison-Wesley, (1996).

28. Libkin, L.: "Incomplete data: what went wrong, and how to fix it." In Proceedings of the 33rd ACM SIGMOD-SIGACT-SIGART symposium on Principles of database systems, 1-13. ACM, (2014).

29. Abiteboul, S., Hull, R., Vianu, V.: Foundations of Databases. Addison-Wesley, (1995).

30. Grahne, G.: The Problem of Incomplete Information in Relational Databases. Springer, (1991).

31. Reiter, R.: On closed world data bases. Logic and Data Bases, 5576, (1977).

32. Reiter, R.: Towards a logical reconstruction of relational database theory. On Conceptual Modelling, 191233, (1982).

33. Libkin, L.: A semantics-based approach to design of query languages for partial information. Semantics in Databases, LNCS 1358, 170208. Springer, (1995).

34. Buneman, P., Jung, A., Ohori, A.: Using power domains to generalize relational databases. TCS, 91(1), 2355, (1991).

35. Deshpande, O., Lamba, D. S., Tourn, M., Das, S., Subramaniam, S., Rajaraman, A., Doan, A.: Building, maintaining, and using knowledge bases: A report from the trenches. In Proceedings of ACM SIGMOD International Conference on Management of Data, 1209-1220, ACM (2013).

36. Dallachiesa, M., Ebaid, A., Eldawy, A., Elmagarmid, A., Ilyas, I. F., Ouzzani, M., Tang, N.: NADEEF: a commodity data cleaning system. In Proceedings of the 2013 ACM SIGMOD International Conference on Management of Data, 541-552, ACM (2013, June). 


\section{Table of Contents}

Determining Query Readiness for Structured Data .............. 1

Farid Alborzi, Rada Chirkova, Jon Doyle, and Yahya Fathi

1 Introduction................................. 1

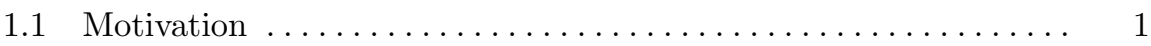

1.2 Contribution and Plan of the Paper $\ldots \ldots \ldots \ldots \ldots \ldots \ldots \ldots \ldots$

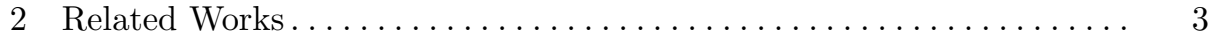

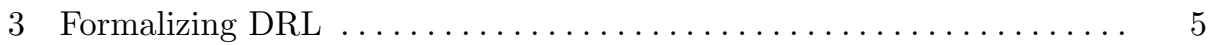

3.1 Example 1: Traffic Flow Identification............... 5

3.2 Tasks . . . . . . . . . . . . . . . . . . . . . . . . . 6

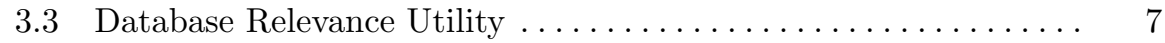

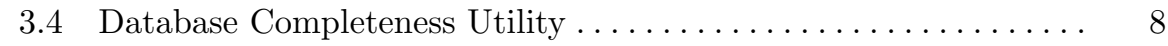

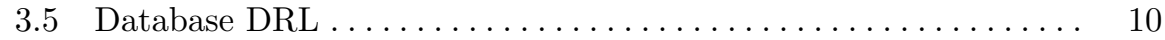

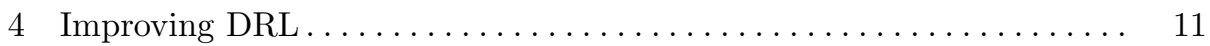

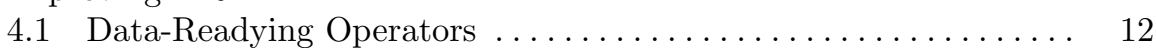

4.2 Null Values Cleaning Operators ..................... 13

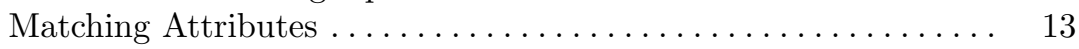

Replacement With the Mean ................... 13

Inferring Values Through Attribute Dependencies . . . . . . . . 14

Evaluating the Range of Null Values . . . . . . . . . . . . . . . . . . . . . . . . . . 14

4.3 Resource Changing Operators . . . . . . . . . . . . . . . . . . 14

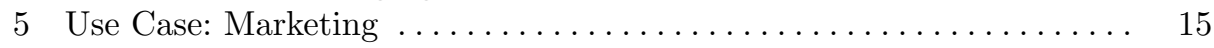

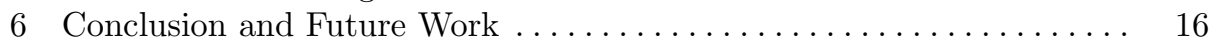

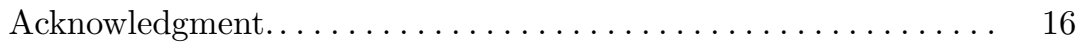

